\title{
Electronic textiles based wearable electrotherapy for pain relief
}

\author{
Meijing Liu ${ }^{1}$, Tyler Ward ${ }^{1}$, Dan Young ${ }^{2}$, Helga Matos ${ }^{1}$, Yang Wei ${ }^{3}$, Jo Adams ${ }^{4}$, Kai Yang ${ }^{1, *}$ \\ ${ }^{1}$ School of Electronics and Computer Science, University of Southampton, SO17 1BJ, Southampton, \\ UK \\ ${ }^{2}$ Hampshire Wellbeing Centre, SO30 3XH, Southampton, UK \\ ${ }^{3}$ School of Science and Technology, Nottingham Trent University, NG11 8NS, Nottingham, UK. \\ ${ }^{4}$ Health Sciences, University of Southampton, SO17 1BJ, Southampton, UK \\ * Email: ky2e09@soton.ac.uk; Tel: +44 (0)23 80596665.
}

\section{Graphical abstract}

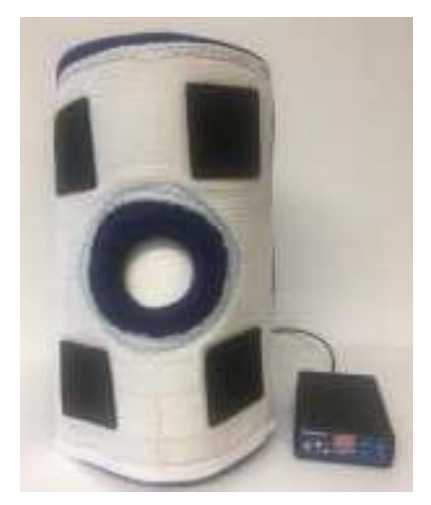

Electrotherapy device

\section{Highlights}

- A fabric electrode has been fabricated using the combination of weaving and printing technologies.

- The conductive yarn pattern was optimized $(2.5 \mathrm{~mm}$ by $2.5 \mathrm{~mm}$ grid) to achieve even current distribution of the electrode layer.

- Asymmetric centrifugal mixing can breakdown the carbon particles and produce a high density (without voids) electrode.

- The wearable electrotherapy was comfortable to wear and easy to use.

- The prototype has been tested on six volunteers with osteoarthritis knee joint pain. Four out of six have reported noticeable pain reduction by using the device. 


\begin{abstract}
Electrotherapy is a common therapeutic treatment used to provide pain relief. The device delivers a mild level of current via electrodes positioned on the skin to interfere with the pain signal and stimulate the release of the body's own natural painkiller to reduce the pain. This paper presents the materials and fabrication methods used to manufacture a textile based wearable electronic textile (e-textile) with electrodes embedded for joint pain relief. The electrode is made by three functional layers including textile layer, conductive layer, and electrode layer. An electronic control has been developed to deliver interferential therapy. The e-sleeve has been designed and developed alongside patient and public input and tested on six volunteers with self-reported knee joint pain. Four out of six volunteers reported noticeable pain reduction on using the e-textile. The wearable e-textile demonstrated no adverse effects and pilot evidence suggests this has the potential to be a comfortable and easy to use solution for pain relief for people living with osteoarthritis knee joint pain.
\end{abstract}

Keywords: Electronic textiles (e-textiles), wearable, electrotherapy, joint pain, osteoarthritis

\title{
1 Introduction
}

Osteoarthritis (OA) is the most common musculoskeletal (MSK) disorder affecting 8.75 million people in the UK and 250 million people worldwide $[1,2]$. OA is a joint disease caused by the structural alterations of the articular cartilage that leads to destruction and failure of the synovial joint. It is an active dynamic alternation due to the imbalance between wear-and-tear and repair. The risk factors of OA include age, obesity, gender (more women than men are affected), and previous knee injury [3]. OA can affect many joints (e.g. knee, hip, hand, feet) and the knee is the most prevalent affected joint due to the regular flexing, bending and twisting, and weight bearing. More than half of the population with OA experiences knee problem with the common symptoms of pain, stiffness, swelling, limited movement in joints, and muscle loss. The number of people living with knee OA in the UK is estimated to increase, with increasing longevity and obesity, from 4.7 million in 2010, to 6.5 million by 2020 and 8.3 million by 2035 [4].

Joint pain is the most disabling symptom that affects people's quality of life and creates a huge burden to healthcare providers and society (e.g. work loss, premature retirement) [1]. Patient information to enhance understanding of the condition and its management, exercise and weight loss have been identified as core treatments to all people with OA [5]. However, joint pain continues to be a significant factor in effective self-management and can impact on the amount of exercise and functional movement a person engage with. .

Electrotherapy devices deliver a mild level electric current (e.g. 0-100 mA) through the skin to interfere the transmission of pain signals by exciting sensory nerves and stimulate the production of endorphins - the bodies' natural painkiller [6,7]. The most commonly used electrotherapy is Transcutaneous Electrical Nerve Stimulation (TENS) that utilizes low frequency (e.g. 2-150 Hz) current. The more advanced electrotherapy is Interferential Current therapy (IFC) that utilises two medium frequency currents (e.g. 1-10 kHz) passing through the tissues at the affected area simultaneously to reduce the pain. IFC eliminates the discomfort and other side effects (e.g. skin irritation) that may be associated with TENS because the skin impendence is significantly reduced at higher frequency (e.g. $3200 \Omega$ at 50 $\mathrm{Hz}$ vs $40 \Omega$ at $4000 \mathrm{~Hz}$ ) [8]. Interferential Current therapy's current can reach greater depths and offer electrotherapy to a larger number of tissues than TENS. Some studies find both TENS and IFC improved pain and functional outcomes without a statistical difference between them [9], while others find IFC is more effective, for example, systematic reviews and meta-analysis have concluded that IFC is an effective treatment to reduce pain, leading to decreasing use of paracetamol [10] and is more effective than other alternative therapeutics (e.g. TENS) [11, 12].

Electrotherapy devices consists of two parts including electronic control unit to generate the current and electrodes to deliver the current through the skin to reach the sensory nerves. Traditional devices that have used sticky gel electrodes having a limited lifetime. The gel electrodes performance reduces as the 
moisture evaporate. Additionally the electrodes are easily contaminated due to the stickiness of the surface property. For the traditional electrotherapy devices, the individual electrodes need to be positioned accurately to optimize performance and this could be time consuming and dependent on operator skill to achieve the correct placement. Wearable TENS devices (e.g. Quell [13], Hollywog [14]) have been developed by integrating the electrodes and electronic control into a single unit to improve the usability. However, the gel electrodes have a limited lifetime typically lasting from 2 to 4 weeks.

To eliminate the disadvantages of gel electrodes, this current work presented here has developed an etextile based sleeve using a novel dry electrode. The soft and tacky (but not sticky) properties of the novel electrode, which distinguishes it from published textile electrodes used in electrical stimulation $[15,16,17]$, provide a comfortable solution to the end users because it combines the advantages of the dry electrode (e.g. easy to use, easy to maintain) and gel electrode (e.g. good skin contact, no hot spots). The electrodes are embedded in a wraparound knee sleeve that is easy to put on and take off. The esleeve has good flexibility and conformability. The electrode material used in this work has passed the cytotoxicity test [18]. It can withstand regularly bending in everyday use and can be cleaned either using wiping or washing [19]. The prototype shown in Figure 1 has been manufactured and tested on six volunteers with OA knee joint pain.

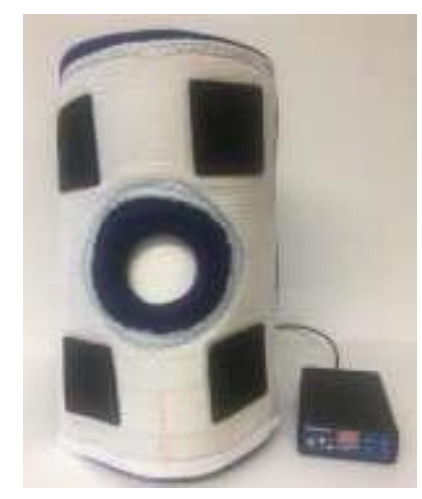

Figure 1. E-sleeve (inside out to show the electrodes) and wired electronic control unit

\section{Material, fabrication and testing methods}

\subsection{Material}

The textile yarn Tencel 2/20 was purchased from Handweavers Studio, UK. The Shieldex ${ }^{\circledR}$ conductive yarn 110/34 dtex 2-ply HC was purchased from Statex, Germany. The knee sleeve (patella design, universal size) was purchased from NEO-G, UK. The electrode pastes Fabink TC-E0002, made of conductive carbon particles and silicone rubber, was supplied by Smart Fabric Inks Ltd, UK. 


\subsection{Fabrication}

\subsubsection{E-sleeve fabrication}

The conductive textile was made by weaving the textile yarn, conductive yarn and encapsulated conductive wire on a Toika electric handloom (Figure 2).

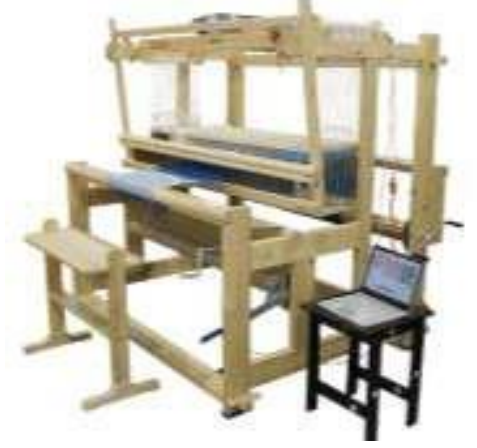

Figure 1. Toika electric handloom

The fabric electrode was fabricated following the process shown in Figure 3. WeavePoint 9 software was used to design the fabric and operate the loom. The fabric consists of two cloths and was woven simultaneously to produce a plain weave structure of a two-layered textile. The bottom layer was made of Tencel yarns which served as a barrier layer to provide protection and electrical insulation. The top layer was made by the combination of Tencel yarn, conductive yarn and silk coated multistrand Litz wire [20], creating the conductive pattern as shown in Figure $\beta$. The size of the conductive pattern was $4 \mathrm{~cm}$ by $4 \mathrm{~cm}$. Different gaps between the conductive yarns in the grid structure were evaluated as detailed in Section 3.1. The encapsulation layer of Litz wire was removed at both ends to provide electrical connection with the conductive yarn and the external electronic control. The electrode was printed on top of the conductive grid using stencil printing (casting the paste through a $1 \mathrm{~mm}$ alumina frame with an open area of $5 \mathrm{~cm} * 5 \mathrm{~cm}$ ) and cured at $80^{\circ} \mathrm{C}$ for $30 \mathrm{~min}$ in a box oven to produce the final fabric electrode.

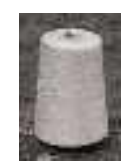

Tencel yarn

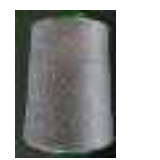

Conductive yarn

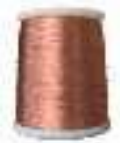

Silk coated wire

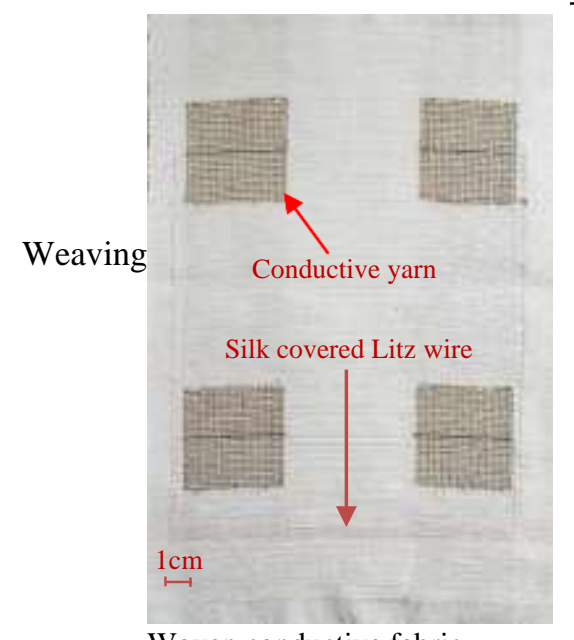

Woven conductive fabric

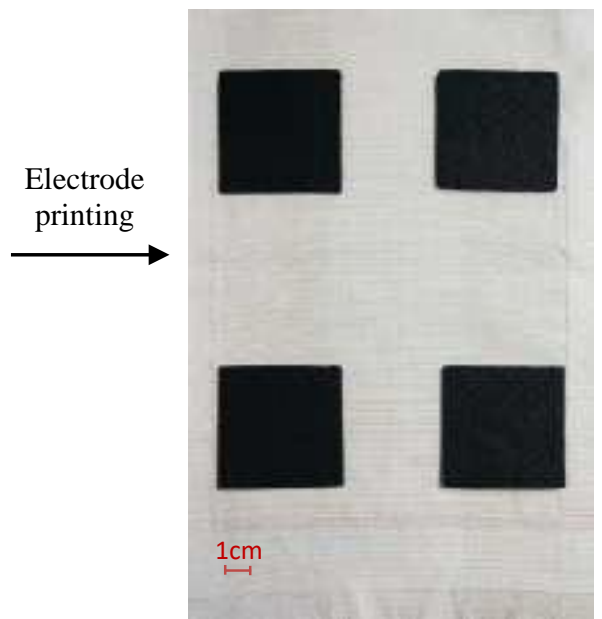

Final electrode

Figure 3. E-sleeve manufacturing

The printed electrode was sewn into the NEO-G knee sleeve to produce the e-sleeve as shown in Figure 4. The interconnection between the conductive wires connected to the electrode and the electronic control was placed on the other side of the knee sleeve as shown in Figure 5. 


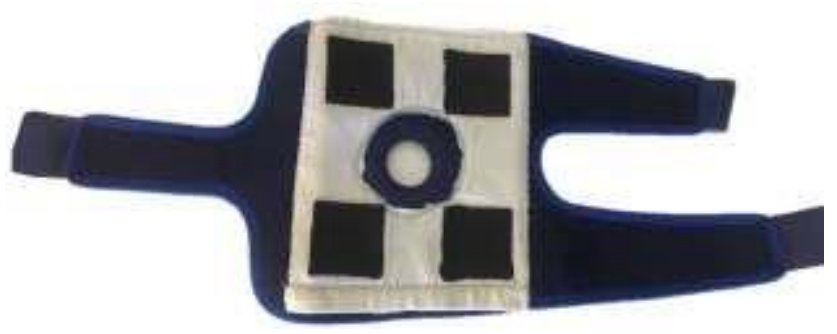

Figure 4. Electrodes on knee sleeve (e-sleeve)

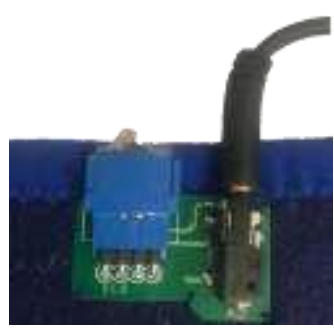

Figure 5. Electrodes and electronnics interconnection

\subsubsection{Electronics}

The electronic control unit provides two separate stimulation currents to the diagonally opposite electrode pairs. Each of the two channels' outputs a stimulation current with a sine wave pattern at a different frequency (one at $4000 \mathrm{~Hz}$ and the other at $4100 \mathrm{~Hz}$ ). The diagonally opposite electrodes (Figure 6) have overlapping current paths inside the tissues of the user. When the two stimulation currents overlap they combine to produce an amplitude modulated signal with a $100 \mathrm{~Hz}$ beat frequency on a $4050 \mathrm{~Hz}$ carrier wave as shown in Figure 7. This waveform is felt as if it is a demodulated $100 \mathrm{~Hz}$ waveform but due to the higher frequency property of the current it can reach deeper into the tissue than using a $100 \mathrm{~Hz}$ wave directly.

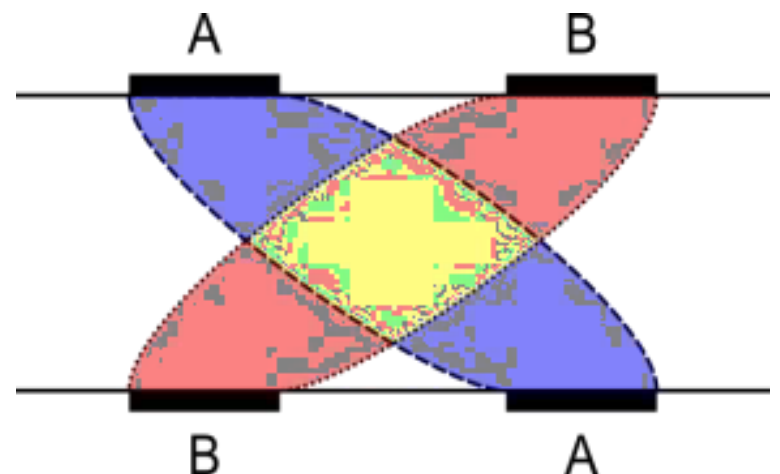

Figure 2. Crossed current paths used in the IFC simulation

The stimulator consists of four subsystems, the battery and power supply, user interface, control processor, and the output stage. The current strength of the stimulation is set on the user interface located on the front of the unit. The control processor then generates an analogue voltage control waveform for each of the two output channels based on the selected strength. The output stage takes the waveforms from the control processor and converts them into current outputs which flow between the two electrodes for channel A and B. The power supply subsystem provides power to the control processor and output stage from the onboard battery and also handles recharging of the battery between uses. The 
electronic was developed according to the safety standard BS EN 60601-2-10 (particular requirements for safety for muscle and nerve stimulation device).

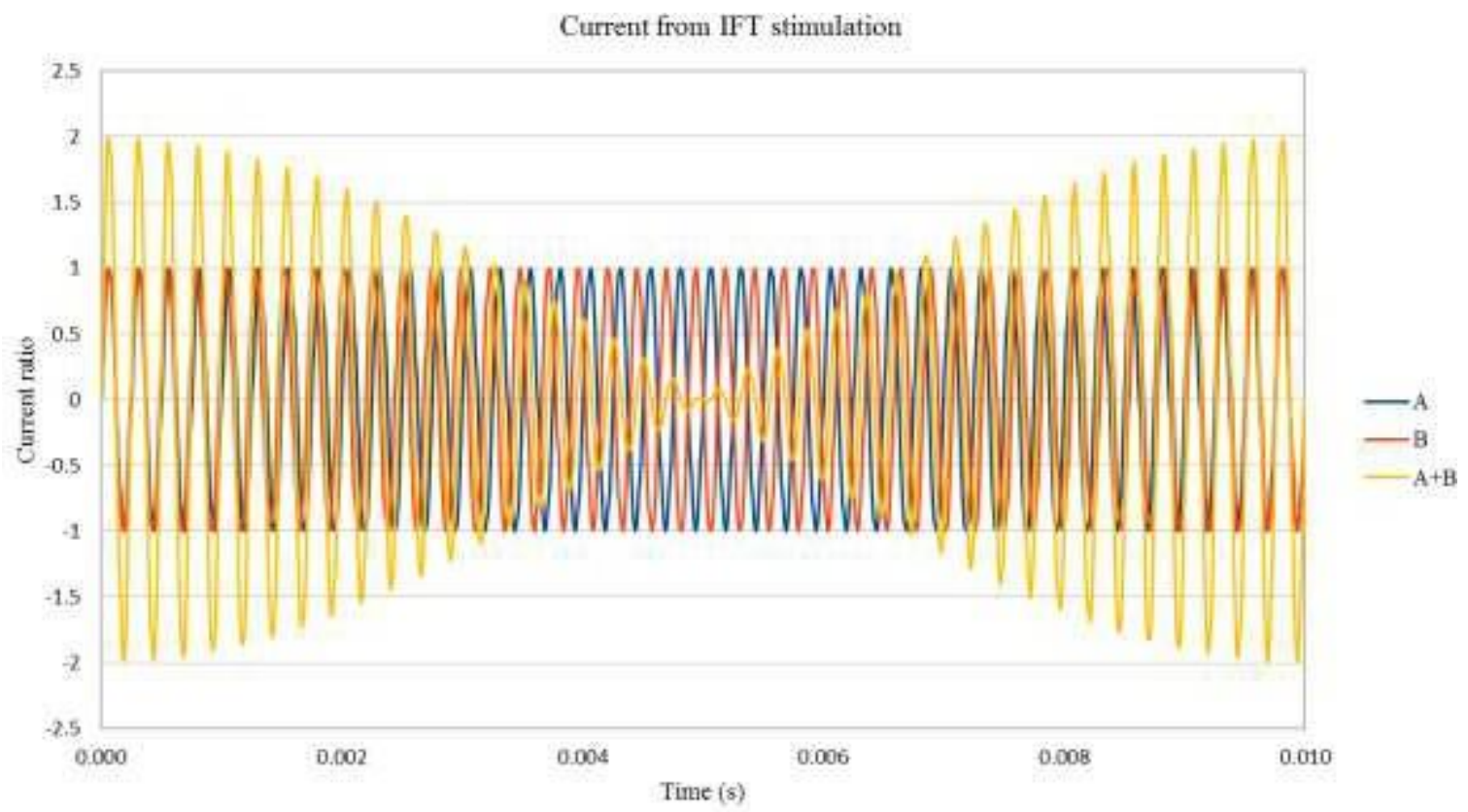

Figure 7. Stimulation currents and merged stimulation effect

\subsection{Testing methods}

\subsubsection{Electrical resistivity test}

The electrical resistance of the carbon electrode was measured by placing it between two conductive plates as shown in the Figure 8. The resistance was measured using a multimeter. The resistance of plates has been deducted in the calculation.

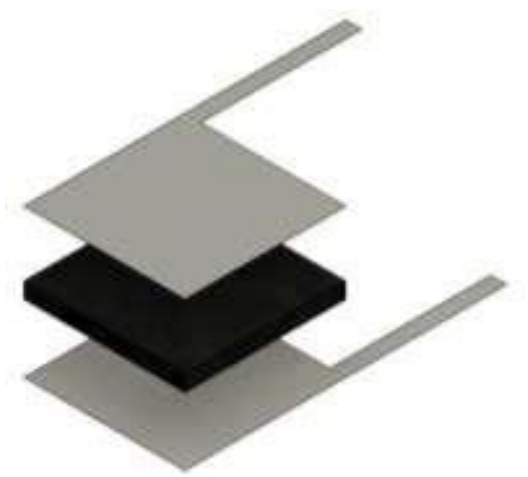

Figure 8. Electrode resistance measurment

The resistivity ( $\rho$ ) of electrode can be calculated using the Equation 1, where R is the resistance, $\mathrm{S}$ is the area of the cross section, and $\mathrm{L}$ is the thickness of the electrode.

$\rho=\mathrm{R} \cdot \mathrm{S} / \mathrm{L}$ 


\subsubsection{Current distribution test}

The current distribution of the electrodes was tested using bespoke equipment. A heatmap was used to represent the current distribution as detailed in Section 3.1. The current was applied to the carbon electrode layer via a pigtail connector which was connected to the conductive yarn layer under the carbon electrode. The current then flows via nine conductive mental pins placed on top the electrode as illustrated in Figure 9.

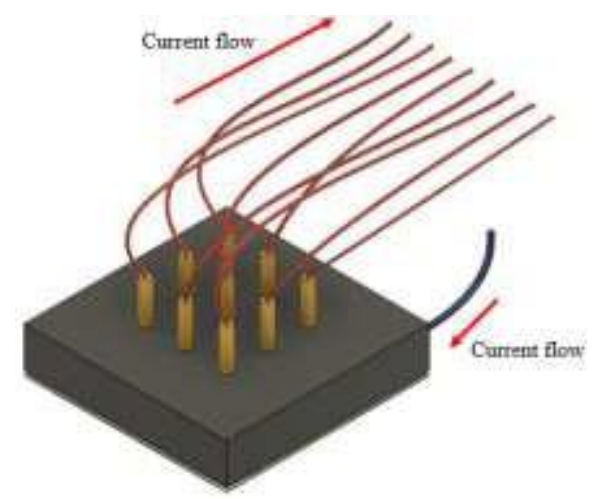

Figure 9. Illustration of the current distribution test

\subsubsection{Prototype testing}

Usability testing of the prototype was conducted with six volunteers (age 50 - 65, 2 female, 4 male) with self-reported knee joint pain recruited from non-NHS routes and not recruited via NHS out-patient clinics or GP surgeries. Full ethical approval from the University of Southampton was gained before conducting any usability testing.

The volunteers were asked to rate their current level of pain prior to testing at rest using a pain scale (Table 1). They were then asked to wear the prototype and adjust the current to the level they felt was strong but not uncomfortable. The prototype was kept on the knee for half hour during a range of activities including walking on flat road, up/down hills, and up/down stairs. Information was collected via verbal feedback on design, pain score during wearing the prototype, and how the prototype might be improved in the future research and development.

Table 1. Pain scale

\begin{tabular}{|c|c|c|c|c|c|c|c|c|c|c|}
\hline $\begin{array}{l}0 \\
\text { Pain Free }\end{array}$ & 1 & 2 & $\begin{array}{l}3 \\
\text { Tolerable }\end{array}$ & $\begin{array}{l}4 \\
\text { Distressing }\end{array}$ & $\begin{array}{l}5 \\
\text { Very } \\
\text { Distressing }\end{array}$ & $\begin{array}{l}6 \\
\text { Intense }\end{array}$ & $\begin{array}{l}7 \\
\text { Very } \\
\text { Intense }\end{array}$ & $\begin{array}{l}8 \\
\text { Utterly } \\
\text { Horrible }\end{array}$ & $\begin{array}{l}9 \\
\text { Excruciating } \\
\text { Unbearable }\end{array}$ & $\begin{array}{l}10 \\
\text { Unimaginable } \\
\text { Unspeakable }\end{array}$ \\
\hline No Pain & \multicolumn{3}{|c|}{ Minor Pain } & \multicolumn{3}{|c|}{ Moderate Pain } & \multicolumn{4}{|c|}{ Severe Pain } \\
\hline $\begin{array}{l}\text { Feeling } \\
\text { perfectly } \\
\text { normal }\end{array}$ & \multicolumn{6}{|c|}{$\begin{array}{l}\text { Nagging, annoying, but doesn'tInterferes significantly with dail } \\
\text { interfere with most daily activities. living activities. Requires lifestyl } \\
\text { Patient able to adapt to painchanges but patient remain } \\
\text { psychologically and with medication } \\
\begin{array}{ll}\text { or devices such as cushions. } & \text { pain. }\end{array} \\
\text { paindent. Patient unable to adapt } \\
\end{array}$} & \multicolumn{4}{|c|}{$\begin{array}{l}\text { Disabling, unable to perform daily living activities. } \\
\text { Unable to engage in normal activities. Patient is } \\
\text { disabled and unable to function independently. }\end{array}$} \\
\hline
\end{tabular}

\section{Results and discussion}

\subsection{The effect of the conductive yarn density on electrode current distribution}

Uniform current distribution on the electrode during the electrotherapy treatment is essential to avoid hot spots that may cause discomfort and pain. The influence of the conductive yarn layer on the electrode current distribution was evaluated by measuring the current distribution among nine points on 
the electrode. Four conductive yarn patterns with a conductive grid size of $5.0 \mathrm{~mm}, 2.5 \mathrm{~mm}, 1.0 \mathrm{~mm}, 0$ $\mathrm{mm}$ as shown in Table 2 were evaluated.

To quantify the difference in current distribution, heatmaps were used to indicate the current distribution among the nine pins. Different colour represents different level of current (green: low; orange: medium; red: high). The heatmaps indicate that electrodes with conductive yarn grid of $2.5 \mathrm{~mm}$ and $1.0 \mathrm{~mm}$ give more uniform current distribution compared to the samples with grid gaps of $5.0 \mathrm{~mm}$ and $0 \mathrm{~mm}$. The average standard deviation $(\sigma)$ in Table 2 confirms the samples with grid gap of $5.0 \mathrm{~mm}$ has poorest current distribution, followed by the samples with $0 \mathrm{~mm}$ (solid conductive yarns). The current distribution for the samples with $1.0 \mathrm{~mm}$ grid gaps were very close to the samples with $2.5 \mathrm{~mm}$ gaps. Therefore, $2.5 \mathrm{~mm}$ gap was used as optimised gap for the conductive yarn layer.

Table 2. Conductive yarn layers with different gird density

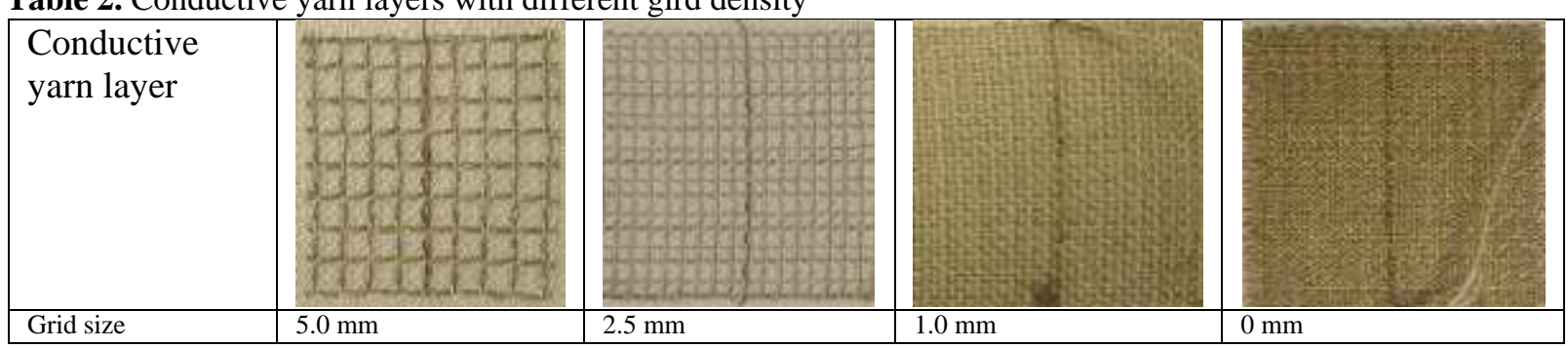

\begin{tabular}{|c|c|c|c|c|c|c|c|c|c|}
\hline \multirow{5}{*}{$\begin{array}{l}\text { Grid size } \\
5.0 \mathrm{~mm}\end{array}$} & \multicolumn{9}{|c|}{ No. of sample tested } \\
\hline & \multicolumn{3}{|l|}{1} & \multicolumn{3}{|l|}{2} & \multicolumn{3}{|l|}{3} \\
\hline & 1.6045 & 1.7477 & 18663 & 1.7083 & 19243 & 1.8476 & $\overline{1.6655}$ & $\longdiv { 1 . 7 1 }$ & 1.8352 \\
\hline & 1.7818 & 19342 & 1.9998 & 1.847 & 1.9506 & 1.9178 & 1.5905 & 1.8386 & 1.7839 \\
\hline & 1.855 & 20299 & 1.8878 & 19659 & 19822 & 1.7941 & 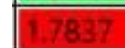 & 1947 & 1.9249 \\
\hline Mean & \multicolumn{3}{|l|}{1.8763} & \multicolumn{3}{|l|}{1.8820} & \multicolumn{3}{|l|}{1.7866} \\
\hline $\begin{array}{l}\text { Standard } \\
\text { deviation }\end{array}$ & \multicolumn{3}{|l|}{0.0851} & \multicolumn{3}{|l|}{0.0452} & \multicolumn{3}{|l|}{0.0616} \\
\hline$\sigma$ & \multicolumn{9}{|c|}{0.06397} \\
\hline \multirow[t]{3}{*}{$2.5 \mathrm{~mm}$} & $\longdiv { 1 . 7 2 1 1 }$ & 1.7241 & 1.7406 & 1.766 & 1.7487 & 1.8275 & 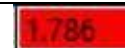 & 1.8623 & $\longdiv { 1 . 8 5 2 }$ \\
\hline & 1.7157 & 1.7115 & 1.7715 & 1.7465 & 1.8622 & 1.7533 & 1.7633 & 1.7802 & 1.8471 \\
\hline & 1.7072 & 1.7127 & 1.7285 & 1,7086 & 1.8338 & 17951 & 1.7079 & 1.6643 & 1.7646 \\
\hline Mean & \multicolumn{3}{|l|}{1.7259} & \multicolumn{3}{|l|}{1.7824} & \multicolumn{3}{|l|}{1.7697} \\
\hline \multirow{2}{*}{$\begin{array}{l}\text { Standard } \\
\text { deviation }\end{array}$} & \multicolumn{3}{|l|}{0.0109} & \multicolumn{3}{|l|}{0.0266} & \multicolumn{3}{|l|}{0.333} \\
\hline & \multicolumn{9}{|l|}{0.0236} \\
\hline \multirow[t]{3}{*}{$1.0 \mathrm{~mm}$} & $\longdiv { 1 . 7 9 5 6 }$ & 1.7741 & 18.8188 & 1.7778 & $\longdiv { 1 . 8 3 4 9 }$ & 1.783 & $\overline{7.8287}$ & 1.8538 & 1.8166 \\
\hline & 18018 & 1.7832 & 1,7985 & 1.7155 & 1.7865 & 1.8351 & 1.763 & 1.8938 & 18575 \\
\hline & 1.8423 & 1.7256 & 1.7358 & 1.8172 & 1.9046 & 1.7402 & 1.807 & 1.9053 & 1.7497 \\
\hline Mean & \multicolumn{3}{|l|}{1.7862} & \multicolumn{3}{|l|}{1.7994} & \multicolumn{3}{|l|}{1.8306} \\
\hline \multirow{2}{*}{$\begin{array}{l}\text { Standard } \\
\text { deviation }\end{array}$} & \multicolumn{3}{|l|}{0.0196} & \multicolumn{3}{|l|}{0.0295} & \multicolumn{3}{|l|}{0.0275} \\
\hline & \multicolumn{9}{|l|}{0.0255} \\
\hline \multirow[t]{3}{*}{$0 \mathrm{~mm}$} & 1.593 & 1.6995 & 1.7474 & 1.6574 & 1.7723 & 1.7701 & 1.7953 & 18602 & 1.8555 \\
\hline & 1.7065 & 1.87 & 1.7701 & 1.6549 & 7.8511 & 1.7815 & 1.6965 & 1.7769 & 1.71 \\
\hline & 1.6239 & 17318 & 1.8299 & 1.6561 & 1.8296 & 1.7122 & 1.7123 & 1.7633 & 1.7247 \\
\hline Mean & 1.7302 & & & 1.7428 & & & 1.7660 & & \\
\hline Standard & 0.0483 & & & 0.041 & & & 0.0329 & & \\
\hline & 0.0407 & & & & & & & & \\
\hline
\end{tabular}




\subsection{Influence of the electrode paste mixing process}

The Fabink TC-E0002 electrode was supplied by three parts: polymer part A, polymer part B, and carbon powder. The percentage of the ingredients recommended by the supplier is $43.5 \%$ part A, $43.5 \%$ part B and $13.0 \%$ carbon. Three different mixing methods were evaluated including hand mixing using spatula, mechanical mixing using KitchenAid mixer, and Dual Asymmetric Centrifugal mixing using SpeedMixer DAC 150. The SEM images of the electrodes cross section were taken using EVO50SVP supplied by Carl Zeiss Ltd. As shown in Figure 10, both hand mixing and KitcheAid mixing introduced air bubbles into the carbon paste in the mixing process. The air was not able to escape during the curing as the paste is viscous. This leads to the formation of voids in the electrode layer. The voids in the electrode made by KitchenAid In addition, there were large carbon particles in the SEM images that indicates the hand mixing and KitchenAid mixer were not able to break down the carbon particles into smaller uniform size. The electrode made using speed mixing produced the best electrode properties with no large voids and more uniform carbon particular distribution. This is because the DAC speed mixer works by the spinning of a high-speed mixing arm in one direction while the basket rotates in the opposite direction. This unique combination of two contra-rotating movements created strong shear force that can break down the large carbon participles to smaller ones. It also eliminate creating bubbles during mixing. Short mixing time (e.g. $20 \mathrm{~s}$ at $3500 \mathrm{rpm}$ ) is essential in order to minimise the heat created which can speed up the polymerisation of the carbon paste.

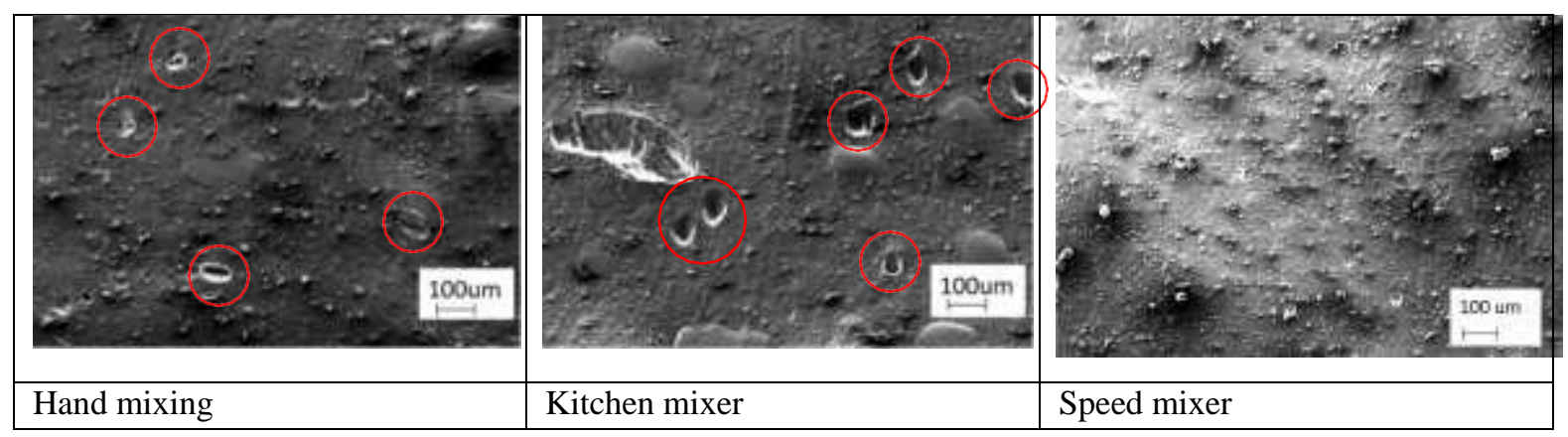

Figure 10. SEM images of the electrodes

\subsection{The influence of external pressure on electrode resistivity}

In order to evaluate the influence of the external pressure on the electrode resistivity, resistance with different weight loading on top of the conductive plate was evaluated from $100 \mathrm{~g}$ to $600 \mathrm{~g}$ with $100 \mathrm{~g}$ intervals, which is equal to adding pressure from $392 \mathrm{~N} / \mathrm{m}^{2}$ to $2352 \mathrm{~N} / \mathrm{m}^{2}$ at $392 \mathrm{~N} / \mathrm{m}^{2}$ intervals. As shown in Figure 11, the resistivity decreased with the increasing of the pressure. In particular, the resistivity reduced by $57 \%$ when the pressure was increased from 0 to $392 \mathrm{~N} / \mathrm{m}^{2}$. This is because the electrode is a soft material which can be squeezed leading to better contact between the carbon particles. Therefore, the e-sleeve should be fit on the knee tightly to ensure good conductivity. 


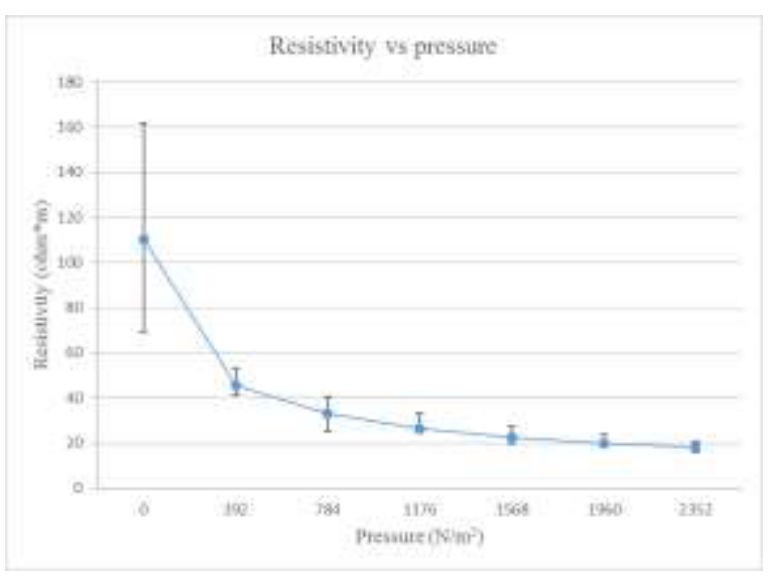

Figure 11. Risistivity under different pressure

\subsection{E-sleeve test}

The electrode fabric was sewn onto an open knee sleeve as shown in Figure 4. The sleeve can be wrapped around the knee and fastened using the Velcro straps to provide tight contact with the skin. The electrodes are connected to the electronic control shown in Figure 12. The current can be adjusted using the push buttons from 0 to $60 \mathrm{~mA}$. The E-sleeve was tested on six volunteers with knee joint pain. The prototype was worn for 30 minutes at the current level the volunteers chosen which was between $30 \mathrm{~mA}$ and $60 \mathrm{~mA}$. The activities carried out with the e-sleeve including walking on flat road, walking up/down hill and walking up/down stairs.

Six out of six volunteers reported the knee sleeve itself (without the IFC on) provided support for the knee. Four out of six reported noticeable pain reduction by using the IFC compared to wearing the knee sleeve without the IFC switched on, in particular for the activities they experienced pain in their everyday life (e.g. walking up and down hill or stairs).

Table 3. Pain scores

\begin{tabular}{|l|l|l|l|}
\hline Participant NO. & Without the e-sleeve & With e-sleeve but without the IFC on & With the e-sleeve and IFC on \\
\hline 1 & $2-3$ & 2 & $0-1$ \\
\hline 2 & 4 & 3 & 3 \\
\hline 4 & 3 & 2 & 2 \\
\hline 5 & $2-3$ & 2 & $0-1$ \\
\hline 6 & 3 & 2 & 1 \\
\hline
\end{tabular}

Six volunteers commented that the stimulation from the e-sleeve was comfortable and the wrap around e-sleeve design was easy to use. The participants were advised to increase the current to maximise the pain relief effect and the current for all participants can reach up 50-60 mA without any discomfort after wearing the e-sleeve for 10 minutes. However, additional feedback on the usability of the wearable technology included to 1) reduce the thickness of the fabric and further improve the flexibility, 2) eliminate the use of the wire cable to improve the user experience. 


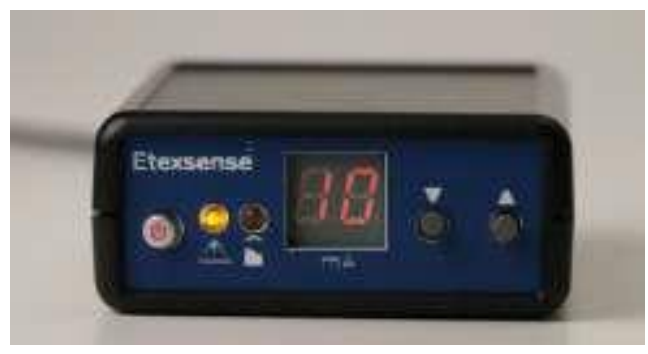

Figure 12. Electronic control

\section{Conclusions}

This paper presents the fabrication of an e-sleeve used for knee joint pain relief. The electrode consists of a textile barrier layer, conductive layer and silicone rubber electrode layer. The conductive pattern with grid size of $2.5 \mathrm{~mm}$ by $2.5 \mathrm{~mm}$ provides a uniform current distribution which eliminates the discomfort that caused by the hot spots of the dry electrodes. Dual Asymmetric Centrifugal SpeedMixer is an effective equipment to mix the electrode paste to reduce the voids and break down large carbon particles. An electronic control system has been developed to provide interferential therapy and the current can be adjusted from 0 to $60 \mathrm{~mA}$ using the push buttons on the interface panel. The prototype has been tested with six volunteers with self-reported knee joint pain and four out of six reported there was noticeable pain reduction on use of the e-sleeve, the stimulation was comfortable and the wraparound e-sleeve was easy to use. Further improvement will be made by using thinner and more flexible fabric to replace the NEO-G sleeve and avoid using the wire cable to improve the use experience.

\section{Funding}

The authors would like to acknowledge funding from EPSRC under grant number EP/S001654/1 and the support from volunteers to test the prototypes.

\section{Declarations of conflicts of interest}

Kai Yang is co-founder of Smart Fabric Inks Ltd.

\section{Acknowledgement}

The authors would like to thank the volunteers for participating the testing.

\section{References}

[1] D. J. Hunter and S. Bierma-Zeinstra, Osteoarthritis, The Lancet, vol. 393, no. 10182, pp. 17451759, 2019.

[2] Arthritis Reserach UK, Arthritis in the UK - facts and statistics, 2014.

[3] D. Prieto-Alhambra, A. Judge, M. K. Javaid, C. Cooper, A. Diez-Perez and N. K. Arden, Incidence and risk factors for clinically diagnosed knee, hip and hand osteoarthritis: influences of age, gender and osteoarthritis affecting other joints, Annals of the rheumatic diseases, vol. 73, no. 9, pp. 1659-1664, 2013. 
[4] Arthritis Research UK, OSTEOARTHRITIS IN GENERAL PRACTICE - Data and perspectives, 2013.

[5] NICE, Management of osteoarthritis, 2019.

[6] S. Guiseppe, J. Ivo, M. Salvatore, B. Angelo and T. Marco, Effect of transcutaneous electrotherapy on CSF $\beta$-endorphin content in patients without pain problems, Pain, pp. 169-172, 1981.

[7] . R. Melzack, Gate control theory: On the evolution of pain concepts, Pain Forum, vol. 5, no. 2, pp. 128-138, 1996.

[8] T. Watson, http://www.electrotherapy.org/modality/interferential-therapy, [accessed on 08 August 2019].

[9] C. C. d. Almeida, V. Z. M. d. Silva, G. C. Júnior, R. E. Liebano and J. L. Q. Durigan, Transcutaneous electrical nerve stimulation and interferential current demonstrate similar effects in relieving acute and chronic pain: a systematic review with meta-analysis, Brazilian journal of physical therapy, vol. 22, no. 5, pp. 347-354, 2018.

[10] M. Buenavente, C. Gonzalez-Suarez, M. Lee-Ledesma and L. Liao, Evidence on the effectiveness of interferential current therapy in the treatment of knee osteoarthritis: A meta-analysis, $O A$ Arthritis, vol. 2, no. 1, pp. 1-9, 2014.

[11] C. Zeng, H. Li, T. Yang, Z. H. Deng, Y. Yang, Y. Zhang and G. H. Lei, Electrical stimulation for pain relief in knee osteoarthritis: systematic review and network meta-analysis, Osteoarthritis and Cartilage, vol. 23, no. 2, pp. 189-202, 2015.

[12] J. Rajfur, M. Pasternok, K. Rajfur, K. Walewicz, B. Fras, B. Bolach, R. Dymarek, J. Rosinczuk, T. Halski and J. Taradaj, Efficacy of Selected Electrical Therapies on Chronic Low Back Pain: A Comparative Clinical Pilot Study, Medical science monitor : international medical journal of experimental and clinical research, vol. 23, pp. 85-100, 2017.

[13] https://www.quellrelief.com/, (accessed on 08 August 2018).

[14] https://www.hollywog.com/, (accessed on 08 August 2018).

[15] K. Gniotek, M. Frydrysiak, J. Zięba, M. Tokarska and Z. Stempień, Innovative textile electrodes for muscles electrostimulation, IEEE International Symposium on Medical Measurements and Applications, pp. 305-310, 2011.

[16] H. Zhou, Y. Lu, W. Chen, Z. Wu, H. Zou, L. Krundel and G. Li, Stimulating the Comfort of Textile Electrodes in Wearable Neuromuscular Electrical Stimulation, Sensors, vol. 15, no. 7, pp. 1724117257, 2015.

[17] A. Ali, V. Baheti, J. Militky and Z. Khan, Utility of silver-coated fabrics as electrodes in electrotherapy applications, Journal of Applied Polymer Science, vol. 135, no. 23, p. 46357, 2018. 
[18] K. Yang, C. Freeman, R. Torah, S. Beeby and J. Tudor, Screen printed fabric electrode array for wearable functional electrical stimulation, Sensors and Actuators A: Physical, vol. 213, pp. 108115, 2014.

[19] K. Yang, K. Meadmore, C. Freeman, N. Grabham, A.-M. Hughes, Y. Wei, R. Torah, M. GlancGostkiewicz, S. Beeby and J. Tudor, Development of User-Friendly Wearable Electronic Textiles for Healthcare Applications, Sensors, vol. 18, no. 8, p. 2410, 2018.

[20] A. Komolafe, R. Torah, Y. Wei, H. Matos, M. Li, D. Hardy, T. Dias, M. Tudor and S. Beeby, Integrating Flexible Filament Circuits for E-Textile Applications, Advanced Materials Technologies, vol. 4, no. 7, pp. 1900176 (1-15), 2019. 


\section{Biographies}

Meijing Liu is currently a $\mathrm{PhD}$ student in Electronics and Computer Science at the University of Southampton. She received her M.S. degree in Microelectromechanical Systems (MEMS) in 2018 from university of Southampton, UK. Her research focuses on advanced E-textiles for healthcare applications.

Tyler Ward graduated with an M.Eng. in Electronics in 2012 from the University of Southampton. He obtained a Ph.D. on internet of things technologies in 2017 also from the University of Southampton. Since completing his $\mathrm{PhD}$, he has been working on turning research ideas into functional electronic systems. One such project he was involved in is Smartmove, which researched the opportunities for the use of functional electrical stimulation combined with computer vision and machine learning for stroke rehabilitation. His research areas include electronic systems, embedded firmware, electrical stimulation systems, and internet of things technologies.

Dan Young is a clinical specialist physiotherapy with an MSc in Sports and Exercise Medicine. He is the clinical lead at the Hampshire Wellbeing Centre. He practices in a busy private clinic in Southampton and has an interest in maintaining physical activity in those affected by pathologies such as osteoarthritis.

Helga Matos is a Specialist Technician of Woven Textiles in the Electronics and Computer Science department at the University of Southampton. She joined the department as part of a EPSRC funded FETT project to integrate electronic strips into woven fabric. She has over 10 years' experience in weaving fabrics for a wide range of uses and projects globally. She gained her Masters degree in constructed Textiles at the Royal College of Art in London where her research focused on studies of materials and fabric construction to create 3D surfaces by weaving.

Yang Wei is a Senior Lecturer in Department of Engineering at Nottingham Trent University. He has also been working in the healthcare technology field where wearable devices are used to measure Electrocardiogram (ECG), Electroencephalogram (EEG), Electromyography (EMG) and respiration. In addition, he has been working on electrical stimulation for stroke patient rehabilitation, deep vein thrombosis (DVT) prevention, and lymphedema management. His research interests include e-textiles, 3D printing, healthcare technologies, sensors and actuators.

Jo Adams is a Professor of Musculoskeletal Health at the University of Southampton. She is a qualified occupational therapist having working clinically and managerially in Health and Social Services before joining academia. Her clinical research background has focused on designing and developing clinical trials that test the effectiveness and efficacy of self- management techniques and devices to improve impairment and function for people living with joint pain and musculoskeletal disorders. She leads initiatives to engage people with musculoskeletal conditions to help develop and design clinical research and has supported NHS clinicians throughout the UK to deliver NHS clinical trials.

Kai Yang is a Principal Research Fellow in the School of Electronics and Computer Science, University of Southampton in UK. She has been awarded an EPSRC Innovation Fellowship in 2018 to develop "Advanced e-textiles for Wearable Therapeutics". She is a Co-I of EPSRC funded E-textiles Network. She was a PI of a Medical Research Council (MRC) Funded SMARTmove project to develop e-textiles based wearable functional electrical stimulation system for stroke upper limb rehabilitation. Her research interests include e-textiles, wearables therapeutics (e.g. rehabilitation, pain relief), ink formulations, printed electronics, electrodes, materials and fabrication. 